\author{
Marquette University \\ e-Publications@Marquette
}

Physical Therapy Faculty Research and

Publications

Physical Therapy, Department of

$12-2017$

\title{
Association Between Sedentary Time and Quality of Life From the Osteoarthritis Initiative: Who Might Benefit Most From Treatment?
}

\author{
Daniel Pinto \\ Marquette University, daniel.pinto@marquette.edu \\ Jing Song \\ Northwestern University \\ Jungwha Lee \\ Northwestern University \\ Rowland W. Chang \\ Northwestern University \\ Pamela A. Semanik \\ Rush University
}

See next page for additional authors

Follow this and additional works at: https://epublications.marquette.edu/phys_therapy_fac

Part of the Physical Therapy Commons

\section{Recommended Citation}

Pinto, Daniel; Song, Jing; Lee, Jungwha; Chang, Rowland W.; Semanik, Pamela A.; Ehrlich-Jones, Linda S.; Pellegrini, Christine A.; and Dunlop, Dorothy D., "Association Between Sedentary Time and Quality of Life From the Osteoarthritis Initiative: Who Might Benefit Most From Treatment?" (2017). Physical Therapy Faculty Research and Publications. 145.

https://epublications.marquette.edu/phys_therapy_fac/145 


\section{Authors}

Daniel Pinto, Jing Song, Jungwha Lee, Rowland W. Chang, Pamela A. Semanik, Linda S. Ehrlich-Jones, Christine A. Pellegrini, and Dorothy D. Dunlop 
Marquette University

e-Publications@Marquette

\section{Physical Therapy Faculty Research and Publications/College of Health Sciences}

This paper is NOT THE PUBLISHED VERSION; but the author's final, peer-reviewed manuscript. The published version may be accessed by following the link in the citation below.

Archives of Physical Medicine and Rehabilitation, Vol. 98, No. 12 (2017): 2485-2490. DOI. This article is (C) Elsevier (WB Saunders) and permission has been granted for this version to appear in ePublications@Marquette. Elsevier (WB Saunders) does not grant permission for this article to be further copied/distributed or hosted elsewhere without the express permission from Elsevier (WB Saunders).

\section{Association Between Sedentary Time and Quality of Life From the Osteoarthritis Initiative: Who Might Benefit Most From Treatment?}

\section{Daniel Pinto}

Department of Physical Therapy and Human Movement Sciences, Feinberg School of Medicine, Northwestern University, Chicago, IL

Jing Song

Department of Preventive Medicine, Feinberg School of Medicine, Northwestern University, Chicago, IL Jungwha Lee

Department of Preventive Medicine, Feinberg School of Medicine, Northwestern University, Chicago, IL Rowland W. Chang Center for Healthcare Studies, Institute for Public Health and Medicine, Feinberg School of Medicine, Northwestern University, Chicago, IL 
Pamela A. Semanik

Rush University, College of Nursing, Chicago, IL

Linda S. Ehrlich-Jones

Center for Rehabilitation Outcomes Research, Shirley Ryan AbilityLab, Chicago, IL

Christine A. Pellegrini

Arnold School of Public Health, University of South Carolina, Columbia, SC

Dorothy D. Dunlop

Center for Healthcare Studies, Institute for Public Health and Medicine, Feinberg School of Medicine, Northwestern University, Chicago, IL

\section{Abstract}

\section{Objective}

To investigate the relationship between sedentary behavior and quality-adjusted life years (QALYs) among participants in the Osteoarthritis Initiative.

Design

Longitudinal, observational design.

\section{Setting}

Osteoarthritis Initiative cohort.

\section{Participants}

Individuals ( $\mathrm{N}=1794)$ from a prospective, multicenter longitudinal cohort were classified into quantile groups based on average daily sedentary time (most sedentary, quartile 1 [Q1] $\geq 11.6 h ; 10.7 h \leq Q 2$ $<11.6 \mathrm{~h} ; 9.7 \mathrm{~h} \leq \mathrm{Q} 3<10.7 \mathrm{~h}$; least sedentary, Q4 <9.7h).

\section{Interventions}

Not applicable.

\section{Main Outcome Measures}

Individual QALYS were estimated over 2 years from the area under the curve of health-related utility scores derived from the Medical Outcomes Study 12-Item Short-Form Health Survey versus time. The relationship between baseline sedentary behavior and median 2-year QALYs was estimated using quantile regression adjusted for socioeconomic factors and body mass index.

Results

Lower QALYs over 2 years were more frequently found among the most sedentary (Q1, median 1.59), and QALYs increased as time spent in baseline sedentary behavior decreased (median QALYs for Q2, 1.64; Q3, 1.65; Q4, 1.65). The relationship of sedentary time and median QALY change was only significant for the most sedentary Q1 group, where an additional hour of sedentary behavior significantly reduced QALYs by -.072 ( $95 \%$ confidence interval, -.121 to -.020 ).

\section{Conclusions}

Our findings suggest that individuals with the most extreme sedentary profiles may be vulnerable to additional losses of quality of life if they become more sedentary. Targeting these individuals to decrease sedentary behavior has the potential to be cost-effective. 


\section{Keywords}

Economics, Osteoarthritis, Observational Study, Quality-adjusted life years, Rehabilitation, Sedentary Lifestyle

\section{List of abbreviations}

$\mathrm{BMI}$, body mass index

MVPA, moderate-vigorous physical activity

OA, osteoarthritis

OAl, Osteoarthritis Initiative

PA, physical activity

Q, quartile

QALY, quality-adjusted life year

SF-6D, Short Form-6 Dimensions

SF-12v2, Medical Outcomes Study 12-Item Short-Form Health Survey, version 2

Adults spend more than half of their waking hours in sedentary behavior ( $<1.5$ metabolic equivalents) including sitting or lying. ${ }^{1}$ Sedentary behavior is associated with poor health outcomes across diseases and an attributable direct medical cost ranging from $\$ 79$ billion to $\$ 131$ billion annually in the United States. ${ }^{2}$ This is a substantial problem in adults with knee osteoarthritis (OA), who spend approximately two thirds of their daily waking time being sedentary. ${ }^{3}$ Consequently, sedentary behaviors have emerged as a separate target for health promotion and disease prevention, distinct from physical activity (PA) promotion. ${ }^{4}$

A limited but growing number of economic evaluations have assessed the cost-effectiveness of PA programs. ${ }^{5}$ The Economics of Physical Inactivity Consensus group recently recommended using the quality-adjusted life year (QALY) as a key endpoint in PA intervention studies. ${ }^{5}$ The QALY is comparable across disciplines and disease states and has broadly accepted levels-for example, $\$ 50,000$ to $\$ 150,000$ per QALY gained in the United States. ${ }^{6}$

Prior work has not formally addressed the cost-effectiveness of sedentary behavior interventions. The purpose of this study was to gain insight into potential target subpopulations for which sedentary behavior interventions are most likely to be a good value. We hypothesized that QALYs are associated with time spent in sedentary behavior and that the strength of the relationship between daily sedentary hours and QALYs would be greatest in the most sedentary subgroups.

\section{Methods}

\section{Participants and setting}

Study participants were part of a subcohort of the Osteoarthritis Initiative (OAI) whose PA behavior was objectively monitored at the OAI 48-month visit. The OAI is a multicenter, longitudinal, prospective observational study of knee OA. The parent OAl study recruited 4796 men and women aged 45 to 79 years with or at an increased risk for developing symptomatic, radiographic knee OA at 4 clinical sites (Baltimore, MD; Columbus, OH; Pittsburgh, PA; Pawtucket, RI) between 2004 and 2006. Radiographic tibiofemoral knee OA was defined as the presence of definite tibiofemoral osteophytes (Osteoarthritis Research Society International atlas grades $1-3,{ }^{7}$ equivalent to Kellgren and Lawrence grade $\left.\geq 2\right)^{8}$ on 
the fixed-flexion radiograph. OAl eligibility criteria have been described in detail elsewhere. ${ }^{9}$ Approval was obtained from the institutional review board at each OAI site and at Northwestern University. Each participant provided written informed consent. The OAI accelerometer ancillary study objectively measured PA from a subcohort of 2127 OAI participants at the OAI 48-month visit, ${ }^{10}$ representing the baseline for the current study. We restricted our sample to 1794 participants with accelerometer monitoring and 2-year follow-up (fig 1). Excluded were 106 nonrespondents/deceased, 168 participants who did not have 4 to 7 valid days (ie, $\geq 10$ wear-hours/d) of accelerometer monitoring, and another 59 participants because of missing data, precluding the calculation of QALYs (see fig 1).

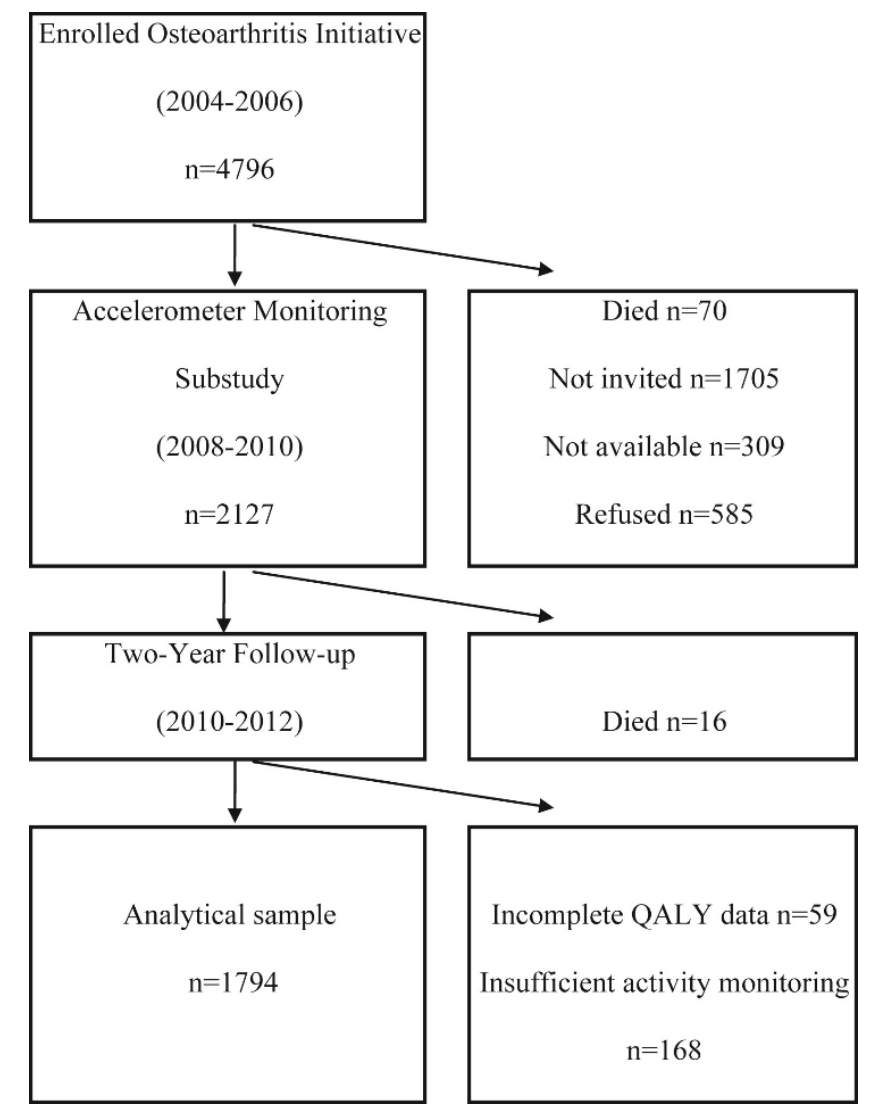

Fig 1. Flow chart of analytical sample.

\section{Outcomes}

PA was objectively measured over the 7 consecutive days after the baseline clinic visit using a GT1M ActiGraph accelerometer, ${ }^{a}$ a small uniaxial accelerometer that measures vertical accelerations. ${ }^{11}$

Trained research personnel gave participants uniform scripted instructions to wear the unit on a belt at the natural waistline on the right hip in line with the right axilla on arising in the morning and continuously until retiring at night, except during water activities. Participants maintained a daily log to record time spent in water and cycling activities, which may not be fully captured by accelerometers. Accelerometer data were analytically filtered using methodology validated in adults with rheumatic disease.12, 13, 14 Nonwear periods were defined as $\geq 90$ minutes with 0 activity counts (allowing for up to 2 consecutive interrupted minutes with counts $<100) .{ }^{13}$ We identified participants with 4 or more valid days (ie, $\geq 10$ wear-hours/d) needed for reliable PA estimates. ${ }^{12}$ Total daily minutes of moderatevigorous PA (MVPA) were calculated using the National Institutes of Health methodology (counts $\geq 2020 / \mathrm{min}$ ). Sedentary behavior, defined by activity counts per minute of $<100$, was used to calculate average daily time spent in sedentary behavior. Minutes of sedentary behavior were translated on a 
minute-by-minute basis from accelerometer output. Sedentary behavior quartiles are based on average daily waking hours spent in sedentary behavior. As performed by Qi et al, ${ }^{15}$ we standardized sedentary time to 16 hours of wear time per day (the typical waking time in our study) because of a high correlation between sedentary time and wear time. The number of standardized sedentary hours per day defined the quartiles bounding quartile 1 (Q1; most sedentary) to quartile 4 (Q4; least sedentary) by 11.6 to $15.1,10.7$ to $11.6,9.7$ to 10.7 , and 4.5 to 9.7 hours, respectively.

Health-related utility was measured using the Medical Outcomes Study 12-Item Short-Form Health Survey, version 2 (SF-12v2) ${ }^{16}$ at baseline and at 2-year follow-up. The SF-12v2 is a 12-dimension instrument for measuring (generic) health-related quality of life that is valid and reliable, ${ }^{17}$ and has excellent test-retest reliability over a 3-week time frame ${ }^{18}$ and good test-retest reliability over a year. ${ }^{17}$ The SF-12 has been abridged to a 6-dimension instrument (SF-6D). ${ }^{19}$ The SF-6D utility is converted from the SF-12 using preference weights estimated from the United Kingdom general adult population. ${ }^{19}$ The SF-6D utility scores range from 0.0 (death, worst health state) to 1.0 (full health, best health state), with an estimated minimally important difference \pm SD of $.027 \pm .028 .{ }^{20}$ An online program (http://www.shef.ac.uk/scharr/sections/heds/mvh/sf-6d) was used to convert baseline and follow-up data on the SF-12v2 to the SF-6D. Participant-level QALYs are typically estimated by weighing each time interval by the individual's utility during that time. Total QALYs are summed by the total of each quality-adjusted time period using the area-under-the-curve method, ${ }^{21}$ which is implemented by summing the areas of the geometric shapes obtained by linear interpolation between utility scores over the study period. ${ }^{22}$ We calculated total QALYs as the geometric shape representing the area under the health-related utility points (ie, the integral) over the course of 2 years. ${ }^{23}$

\section{Covariates}

Covariates included socioeconomic factors and body mass index (BMI). Socioeconomic factors included age, sex, self-reported race/ethnicity (African American, white, or other race), education, marriage status, and income. BMI was calculated from measured height and weight (weight $[\mathrm{kg}] / \mathrm{height}[\mathrm{m}]^{2}$ ). Persons were classified as normal weight (BMI, 18.5-24.9), overweight (BMI, 25.0-29.9), or obese (BMI, $\geq 30)$.

We did not include as covariates health factors represented in the QALY. The decision to leave these covariates out of the model was because of the innate relationship between health factors and the QALY. If adjusted for in the model, the endpoint (health-related quality of life or its surrogates) would be "to some extent represented on both sides of the model equation," ${ }^{24(p .5)}$ which will result in a biased estimate.

\section{Data analysis}

We presented descriptive analyses of participant characteristics by sedentary time in quartiles. We examined the association between baseline sedentary behavior and the QALY by sedentary quartile using quantile regression models, controlling for baseline covariates. Quantile regression was developed to estimate change in the response variable $y$ as a function of predictor variables $x$ when the probability distribution does not follow a parametric distribution.25, 26 Quantile regression differs from ordinary least-squares regression, which estimates the effect as a change in the mean of the distribution of response variable $y .{ }^{26}$ When the probability distribution is nonparametric, estimating a change in the mean becomes problematic, potentially underestimating, overestimating, or failing to distinguish nonzero changes in distributions. The estimates generated using quantile regression are semiparametric in the sense that no parametric distributional form (eg, normal, Poisson, negative 
binomial) is assumed for the random error part of the model, although a parametric form is assumed for the determinist portion of the model. ${ }^{25}$ Quantile regression modeling the median was used in this analysis because the data did not meet a parametric distributional form. Quantile regression is robust to outliers and does not require assumptions regarding the underlining distribution of the outcome to obtain valid inference tests.

We considered the effect modification of sedentary behavior and sedentary time on the QALY to assess whether the effect of sedentary behavior was different across quartiles. We used isotemporal substitution models to estimate the potential benefits gained for substituting sedentary behavior with PA. ${ }^{27}$ The isotemporal substitution model allows us to trade the consequences of alternative PAs to assess the potential benefit from an alternative use of one's time. ${ }^{7}$ The isotemporal substitution model allows us to evaluate the effect of replacing 1 hour of sedentary time with 1 hour of time spent in light PA and MVPA, respectively. In our analyses we examine the "substitution effect" while also adjusting for the independent effects of other covariates simultaneously.

We performed analyses using Stata/SE $13.1^{\mathrm{b}}$ and SAS software version $9.4 .^{\mathrm{c}}$ We set statistical significance at a $P$ level of $\leq .05$.

\section{Results}

The 1794 participants evaluated in this study had a mean age of 65 years (range, 49-83y); 55\% were women, $85 \%$ were white, and $30 \%$ had 1 or more comorbidities. On a daily average, participants spent a mean \pm SD of $10.6 \pm 1.39$ hours in sedentary behavior, $5.07 \pm 1.29$ hours in light PA, and $19.5 \pm 20.38$ minutes in MVPA. Baseline sample characteristics are summarized by sedentary behavior quartile in table 1. The most sedentary group (Q1) was sedentary approximately three fourths of their waking time (ie, $>12.3 \mathrm{~h}$ on average each day of 16 waking hours) and tended to be older, male, ethnically white, and obese, with a lower income than the less sedentary groups.

Table 1. Baseline characteristics by average daily sedentary hours in adults with or at risk for knee OA ( $N=1794)$

\begin{tabular}{|c|c|c|c|c|}
\hline Characteristic & $\begin{array}{c}\text { Q1 (Most } \\
\text { Sedentary, 11.6- } \\
\text { 15.1h; } n=439 \text { ) }\end{array}$ & $\begin{array}{c}\text { Q2 (10.7- } \\
11.6 h ; n=464)\end{array}$ & $\begin{array}{c}\text { Q3 (9.7-10.7h; } \\
n=456)\end{array}$ & $\begin{array}{c}\text { Q4 (Least } \\
\text { Sedentary, 4.5- } \\
\text { 9.7h; } n=435 \text { ) }\end{array}$ \\
\hline \multicolumn{5}{|l|}{ Socioeconomics } \\
\hline Age (y) & $68.8 \pm 9.6$ & $65.5 \pm 8.8$ & $63.7 \pm 8.5$ & $62.3 \pm 7.9$ \\
\hline Female & 45.6 & 53.2 & 57.0 & 63.5 \\
\hline Nonwhite & 13.2 & 14.9 & 13.8 & 19.5 \\
\hline $\begin{array}{l}\text { Education } \leq \text { high school } \\
\text { or not reported }\end{array}$ & 13.2 & 13.2 & 11.0 & 13.3 \\
\hline Income $<\$ 50,000^{*}$ & 39.6 & 29.7 & 28.1 & 31.7 \\
\hline BMI $\left(\mathrm{kg} / \mathrm{m}^{2}\right)$ & $29.2 \pm 5.0$ & $28.6 \pm 4.7$ & $28.1 \pm 4.8$ & $27.9 \pm 4.7$ \\
\hline Normal weight & 22.1 & 23.5 & 29.2 & 28.1 \\
\hline Overweight & 33.9 & 42.7 & 38.2 & 42.1 \\
\hline Obesity & 44.0 & 33.8 & 32.7 & 29.9 \\
\hline \multicolumn{5}{|l|}{ PA profile } \\
\hline Sedentary $(\mathrm{h} / \mathrm{d})$ & $12.30 \pm 0.58$ & $11.12 \pm 0.26$ & $10.21 \pm 0.29$ & $8.75 \pm 0.86$ \\
\hline Light $(\mathrm{h} / \mathrm{d})$ & $3.56 \pm 0.56$ & $4.60 \pm 16.79$ & $5.39 \pm 0.43$ & $6.77 \pm 0.86$ \\
\hline $\begin{array}{l}\text { Moderate/vigorous } \\
(\mathrm{min} / \mathrm{d})\end{array}$ & $8.48 \pm 10.66$ & $16.79 \pm 16.30$ & $23.97 \pm 21.95$ & $28.81 \pm 23.95$ \\
\hline
\end{tabular}

NOTE. Values are mean \pm SD or $\%$. 
$*$ Unknown was combined with $>\$ 50,000$.

Lower QALYs were more frequently found among the most sedentary (Q1, median 1.59), and QALYs increased as time spent in sedentary behavior decreased, with median values of 1.64, 1.65, and 1.65 for Q2, Q3, and Q4, respectively. Figure 2 shows the cumulative frequency curves of QALYs stratified by sedentary quartile. Each point on the graph represents the percentage (vertical axis) of participants within that sedentary group with a QALY score equal to or greater than the value on the $x$ axis. The cumulative frequency of QALYs shows a distinct separation in the direction of lower QALYS (approximately 10\% lower utility) for the curve representing Q1 from the other quartiles over a broad range of QALY values (1.36-1.78). This figure indicates worse QALYs are more frequently found among adults who are the most sedentary.

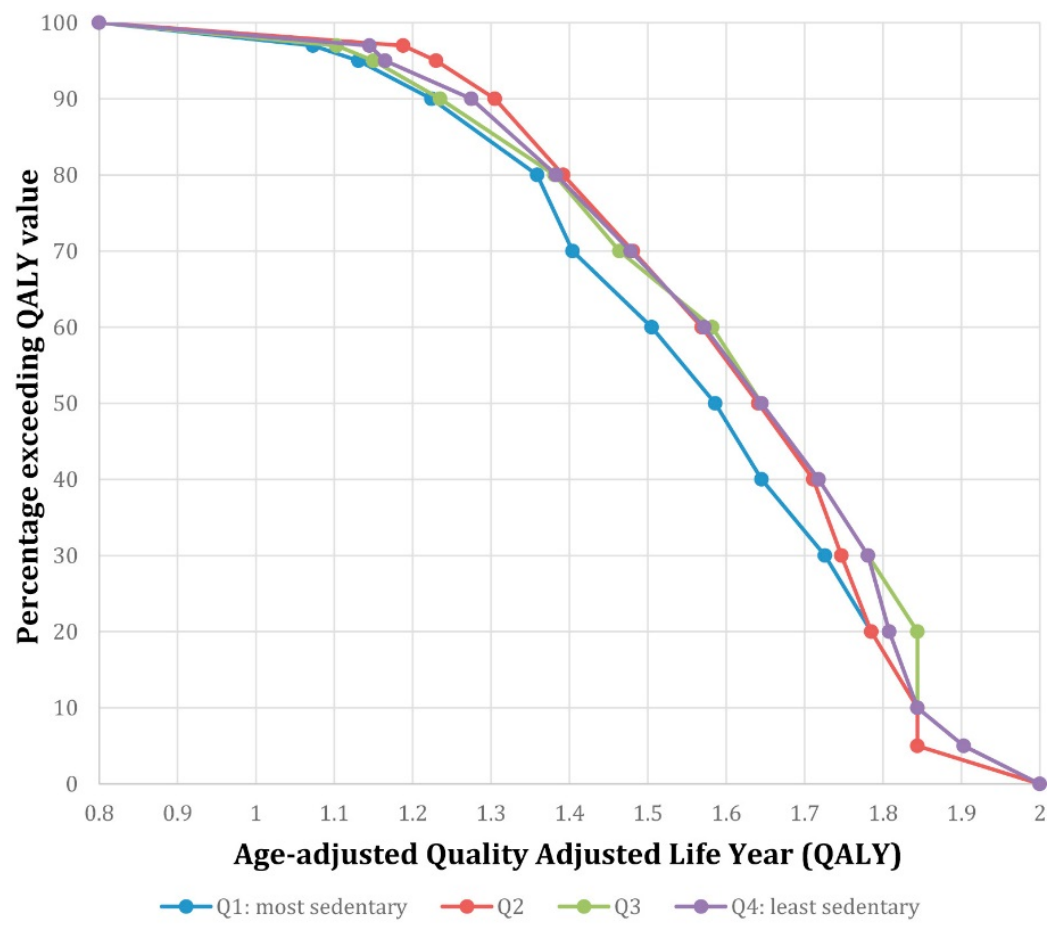

Fig 2. Age-adjusted 2-year QALYs by sedentary quartile groups ( $\mathrm{N}=1794)$.

The effect of time spent in sedentary behavior on QALYs for each quartile group was estimated from interaction terms (sedentary group $\times$ sedentary time) using quantile regression models. Further analyses evaluated the influence of socioeconomic factors and BMI identified in table 1 . Table 2 reports on the relationship between sedentary time and QALYs for each sedentary group. Median QALY change size and significance appear quartile dependent. The most sedentary quartile (Q1) showed a significant QALY loss with an additional hour of sedentary behavior: -.072 (95\% confidence interval, -.125 to -.020$)$. Controlling for sociodemographic factors, marital status, and BMI results in a sizable and significant QALY loss. In contrast, no other quartiles show a significant relationship between QALYs and time spent in sedentary behavior. Recognizing comorbidity may act on the same pathway as PA, a sensitivity analysis further controlled for comorbidities. These results indicated $<30 \%$ of the problematic sedentary behavior effects on QALY were shared with comorbidity for the most sedentary group. 
Table 2. Change $(95 \% \mathrm{Cl})$ in median QALY per additional hour of sedentary behavior using median regression

\begin{tabular}{|c|c|c|c|c|c|}
\hline & Adjustment & $\begin{array}{l}\text { Q1 (Most Sedentary, } \\
\text { 11.6-15.1h; } \mathrm{n}=439 \text { ) }\end{array}$ & $\begin{array}{l}\text { Q2 (10.7- } \\
11.6 h ; \\
n=464)\end{array}$ & $\begin{array}{l}\text { Q3 (9.7- } \\
10.7 \mathrm{~h} ; \\
\mathrm{n}=456)\end{array}$ & $\begin{array}{c}\text { Q4 (Least } \\
\text { Sedentary, 4.5- } \\
9.7 \mathrm{~h} ; \mathrm{n}=435 \text { ) }\end{array}$ \\
\hline \multirow[t]{2}{*}{$\begin{array}{l}\text { Sedentary } \\
\text { behavior }\end{array}$} & Unadjusted & $\begin{array}{l}-.072 *(-.125 \text { to } \\
-.020)\end{array}$ & $\begin{array}{l}.009(-.104 \\
\text { to } .122)\end{array}$ & $\begin{array}{l}.044(-.061 \\
\text { to } .149)\end{array}$ & $.007(-.029$ to .042$)$ \\
\hline & $\begin{array}{l}\text { SES + marital } \\
\text { status + BMI }\end{array}$ & $\begin{array}{l}-.067^{*}(-.120 \text { to } \\
-.014)\end{array}$ & $\begin{array}{l}-.012(-.126 \\
\text { to } .101)\end{array}$ & $\begin{array}{l}.036(-.069 \\
\text { to } .142)\end{array}$ & $.015(-.020$ to .049$)$ \\
\hline
\end{tabular}

Abbreviations: $\mathrm{Cl}$, confidence interval; SES, socioeconomic status. $* p<0.05$.

For individuals within the most sedentary quartile, we performed an isotemporal substitution analysis to understand the potential benefits gained from substituting 1 hour of daily sedentary behavior with 1 hour of light PA or with 1 hour of MVPA. Exchanging sedentary time with 1 hour of light PA (holding MVPA constant and adjusting for sociodemographic and health factors) would result in a median improvement of .03 QALYs over 2 years $(P=.204)$. Exchanging 1 hour of sedentary time for MVPA (holding light PA constant and adjusting for sociodemographic and health factors) would result in a median improvement of .23 QALYs $(P=.05)$. These results suggest that exchanging 10 minutes of sedentary time with MVPA produces similar QALY returns as exchanging 1 hour of light PA.

\section{Discussion}

The main findings of our study showed that only within the most sedentary quartile group did sedentary time and quality of life have a significant detrimental relationship. Our isotemporal analysis allowed us to consider the exchange of sedentary behavior with low and moderate/vigorous activities and the resulting implications on quality of life. Substituting sedentary time in the most sedentary quartile group with either MVPA or light PA could potentially improve QALYs. Our findings suggest that substituting sedentary time with MVPA produces 6 times the QALY gain achieved by substituting sedentary time with light PA for the most sedentary adult.

\section{Implications for clinical practice and designing clinical trials}

This study may help identify individuals who are most likely to benefit from interventions to reduce sedentary behavior when considering the QALY as a unit of outcome. In the most sedentary quartile, an additional hour of sedentary behavior significantly reduced QALYs by .072 QALYs over a 2-year period, yielding an average annual QALY gain of .036. This level of change is greater than the minimum important difference for the SF-6D ${ }^{20}$ and is greater than QALY changes found in economic studies assessing nonsurgical, nonpharmacologic interventions for hip or knee arthritis. ${ }^{28}$

These results may be informative to clinical practice. Our results suggest that equivalent QALY gains can be achieved by replacing sedentary time with either 10 minutes of MVPA or 1 hour of light PA. Interventions can include promoting MVPA or light PA behavior, or both, based on patient preference. While there are challenges to identify the very sedentary individual, ${ }^{29}$ we suggest incremental steps designed to break up sedentary behavior. Australian PA guidelines specifically recommend individuals to "minimize time spent in sedentary behavior every day" and "break up long periods of sitting as often as possible." ${ }^{30(p .4)}$ An incremental approach such as this with small, achievable goals is encouraged and based on behavioral models. ${ }^{31}$ Specific applications include recommending that patients stand during advertising breaks when watching television programs, walk around the couch or chair, or make short trips such as walking to the mailbox. 
These results may also be helpful in clinical trial design. Interestingly, no quartiles other than the most sedentary showed a significant change in QALYs when the effect modification between sedentary time and sedentary behavior was assessed. The whole cohort of individuals with or at risk for knee OA appears heterogeneous with respect to the effect of sedentary behavior on QALYs, suggesting that addressing sedentary behavior in more active individuals is not likely to improve quality of life as captured by the QALY. Likewise, conducting a clinical trial to replace sedentary behavior using the whole cohort may not produce meaningful changes in QALYs. Our findings suggest that researchers assessing strategies to replace sedentary behavior should use caution when selecting their study sample if the QALY is an outcome of interest.

\section{Study strengths and limitations}

Strengths of this study include longitudinal data collection across multiple sites, the large sample size, the age and sex diversity of the sample, and the objective capture of activity behavior using accelerometers. Our study had several limitations. Accelerometers are not sensitive to detect all activities such as cycling or upper body movement, and accelerometers are not worn during waterbased activities (however activity logs indicate little total activity was accounted for by water-based activities in this sample). In our analysis we did not adjust for marital status, which can potentially influence the QALY. As a sensitivity analysis we added marital status to the model as a covariate and found that our results in table 2 remained the same and our isotemporal results for MVPA substitution remained significant (results not shown). The associations found in this study do not necessarily translate to what would be observed if sedentary behavior was changed since they are not necessarily causal.

\section{Conclusions}

Individuals with the most extreme sedentary profiles may be vulnerable to additional losses of quality of life if they become more sedentary. It is likely that meaningful QALY gains are possible if sedentary behavior can be substituted with light PA or MVPA. There exists the potential for a cost-effective intervention reducing sedentary behavior.

\section{Suppliers}

a. GT1M ActiGraph accelerometer; ActiGraph LLC.

b. Stata/SE 13.1; StataCorp LP.

c. SAS software version 9.4; SAS Institute Inc.

\section{References}

1 C.E. Matthews, K.Y. Chen, P.S. Freedson, et al. Amount of time spent in sedentary behaviors in the United States, 2003-2004. Am J Epidemiol, 167 (2008), pp. 875-881.

2 S.A. Carlson, J.E. Fulton, M. Pratt, Z. Yang, E.K. Adams. Inadequate physical activity and health care expenditures in the United States. Prog Cardiovasc Dis, 57 (2015), pp. 315-323.

3 J. Lee, R.W. Chang, L. Ehrlich-Jones, et al. Sedentary behavior and physical function: objective evidence from the Osteoarthritis Initiative. Arthritis Care Res (Hoboken), 67 (2015), pp. 366-373

4 L.H. Epstein, J.N. Roemmich. Reducing sedentary behavior: role in modifying physical activity. Exerc Sport Sci Rev, 29 (2001), pp. 103-108. 
5 J.C. Davis, E. Verhagen, S. Bryan, et al. 2014 consensus statement from the first Economics of Physical Inactivity Consensus (EPIC) conference (Vancouver). Br J Sports Med, 48 (2014), pp. 947-951.

6 P.A. Ubel, R.A. Hirth, M.E. Chernew, A.M. Fendrick. What is the price of life and why doesn't it increase at the rate of inflation? Arch Intern Med, 163 (2003), pp. 1637-1641.

7 R.D. Altman, M. Hochberg, W.A. Murphy Jr., F. Wolfe, M. Lequesne. Atlas of individual radiographic features in osteoarthritis. Osteoarthritis Cartilage, 3 (Suppl A) (1995), pp. 3-70.

8 J.H. Kellgren, J.S. Lawrence. Radiological assessment of osteo-arthrosis. Ann Rheum Dis, 16 (1957), pp. 494-502.

9 M. Nevitt, D. Felson, G. Lester. The Osteoarthritis Initiative protocol for the cohort study. 2006 Available at: http://oai.epi-ucsf.org/datarelease/docs/StudyDesignProtocol.pdf

10 D.D. Dunlop, J. Song, P.A. Semanik, et al. Objective physical activity measurement in the Osteoarthritis Initiative: are guidelines being met? Arthritis Rheum, 63 (2011), pp. 3372-3382.

11 C.E. Matthews, B.E. Ainsworth, R.W. Thompson, D.R. Bassett Jr. Sources of variance in daily physical activity levels as measured by an accelerometer. Med Sci Sports Exerc, 34 (2002), pp. 1376-1381.

12 R.P. Troiano, D. Berrigan, K.W. Dodd, L.C. Masse, T. Tilert, M. McDowell. Physical activity in the United States measured by accelerometer. Med Sci Sports Exerc, 40 (2008), pp. 181-188.

13 J. Song, P. Semanik, L. Sharma, et al. Assessing physical activity in persons with knee osteoarthritis using accelerometers: data from the Osteoarthritis Initiative. Arthritis Care Res (Hoboken), 62 (2010), pp. 1724-1732.

14 P. Semanik, J. Song, R.W. Chang, L. Manheim, B. Ainsworth, D. Dunlop. Assessing physical activity in persons with rheumatoid arthritis using accelerometry. Med Sci Sports Exerc, 42 (2010), pp. 1493-1501.

15 Q. Qi, G. Strizich, G. Merchant, et al. Objectively measured sedentary time and cardiometabolic biomarkers in US Hispanic/Latino adults: the Hispanic Community Health Study/Study of Latinos (HCHS/SOL). Circulation, 132 (2015), pp. 1560-1569.

16 J. Ware Jr., M. Kosinski, S.D. Keller. A 12-Item Short-Form Health Survey: construction of scales and preliminary tests of reliability and validity. Med Care, 34 (1996), pp. 220-233.

17 N.C. Cheak-Zamora, K.W. Wyrwich, T.D. McBride. Reliability and validity of the SF-12v2 in the medical expenditure panel survey. Qual Life Res, 18 (2009), pp. 727-735.

18 R.W. Bohannon, R. Maljanian, M. Landes. Test-retest reliability of short form (SF)-12 component scores of patients with stroke. Int J Rehabil Res, 27 (2004), pp. 149-150.

19 J. Brazier, J. Roberts. The estimation of a preference-based measure of health from the SF-12. Med Care, 42 (2004), pp. 851-859.

20 N. Luo, J. Johnson, S.J. Coons. Using instrument-defined health state transitions to estimate minimally important differences for four preference-based health-related quality of life instruments. Med Care, 48 (2010), pp. 365-371.

21 J.N. Matthews, D.G. Altman, M.J. Campbell, P. Royston. Analysis of serial measurements in medical research. BMJ, 300 (1990), pp. 230-235.

22 A. Manca, N. Hawkins, M.J. Sculpher. Estimating mean QALYs in trial-based cost-effectiveness analysis: the importance of controlling for baseline utility. Health Econ, 14 (2005), pp. 487-496.

23 M.F. Drummond, M.J. Sculpher, G.W. Torrance, B.J. O'Brien, G.L. Stoddart. Methods for the economics evaluation of health care programmes (3rd ed.), Oxford University Pr, New York (2005).

24 C.R. Weinberg. Toward a clearer definition of confounding. Am J Epidemiol, 137 (1993), pp. 1-8. 
25 B.S. Cade, B.R. Noon. A gentle introduction to quantile regression for ecologists. Front Ecol Environ, 1 (2003), pp. 412-420.

26 R. Koenker, G. Bassett. Regression quantiles. Econometrica, 46 (1978), pp. 33-50.

27 R.A. Mekary, W.C. Willett, F.B. Hu, E.L. Ding. Isotemporal substitution paradigm for physical activity epidemiology and weight change. Am J Epidemiol, 170 (2009), pp. 519-527.

28 D. Pinto, M.C. Robertson, P. Hansen, J.H. Abbott. Cost-effectiveness of nonpharmacologic, nonsurgical interventions for hip and/or knee osteoarthritis: systematic review. Value Health, 15 (2012), pp. 1-12.

29 A.L. Gilbert, J. Lee, M. Ma, et al. Comparison of subjective and objective measures of sedentary behavior using the Yale Physical Activity Survey and accelerometry in patients with rheumatoid arthritis. J Phys Act Health, 13 (2016), pp. 371-376.

30 The Department of Health. Australia's physical activity and sedentary behaviour guidelines. Australian Government, Canberra, Aus (2014).

31 N.T. Artinian, G.F. Fletcher, D. Mozaffarian, et al. Interventions to promote physical activity and dietary lifestyle changes for cardiovascular risk factor reduction in adults: a scientific statement from the American Heart Association. Circulation, 122 (2010), pp. 406-441.

Supported in part by the National Institute of Arthritis and Musculoskeletal and Skin Diseases (grant nos. R01-AR054155, R21-AR059412, P60-AR064464); National Center for Advancing Translational Sciences (Clinical and Translational Sciences award no. UL1TR000150); Agency for Healthcare Research and Quality (training grant no. K12HS023011); and the Foundation for Physical Therapy's Center of Excellence in Physical Therapy Health Services and Health Policy Research and Training grant.

Disclosures: none. 